\title{
Differential Effects of Cerebellar Inactivation on Eyeblink Conditioned Excitation and Inhibition
}

\author{
John H. Freeman Jr, Hunter E. Halverson, and Amy Poremba \\ Department of Psychology, University of Iowa, Iowa City, Iowa 52242
}

The neural mechanisms underlying excitatory and inhibitory eyeblink conditioning were compared using muscimol inactivation of the cerebellum. In experiment 1 , rats were given saline or muscimol infusions into the anterior interpositus nucleus ipsilateral to the conditioned eye before each of four daily excitatory conditioning sessions. Postinfusion testing continued for four more excitatory conditioning sessions. All rats were given a final test session after muscimol infusions. The muscimol infusions inactivated the cerebellar nuclei, lateral anterior lobe, crus I, rostral crus II, and lobule HVI ipsilateral to the conditioned eye. Acquisition of excitatory conditioning was completely prevented by muscimol inactivation. In experiment 2 , there were four experimental phases. Phase 1 consisted of excitatory conditioning. In phase 2 , rats were given saline or muscimol infusions before conditioned inhibition training. Phase 3 consisted of continued conditioned inhibition training with no drug infusions. In phase 4, all rats received a retardation test in which the inhibitory stimulus was paired with the unconditioned stimulus. Muscimol infusions blocked the expression of conditioned responses during phase 2. However, robust conditioned inhibition was evident in phases 3 and 4 . The findings indicate that conditioned excitation and inhibition depend on different mechanisms.

Key words: cerebellum; interpositus; learning; conditioning; eyeblink; muscimol

\section{Introduction}

The standard classical conditioning procedure establishes a predictive relationship between a conditioned stimulus (CS) and an unconditioned stimulus (US). After repeatedly pairing the CS and US, a conditioned response (CR) is elicited by the CS. Variants of the standard classical conditioning procedure can be used to produce inhibition of CRs by establishing a second CS as a predictor that the US will be omitted. A commonly used method for establishing inhibitory learning is to present standard CS-US conditioning trials mixed with trials in which the original CS (CSA) is presented with a second CS (CSX) simultaneously, and the compound stimulus is not paired with the US (Pavlov, 1927; Freeman and Nicholson, 1999; Nicholson and Freeman, 2002; Nolan et al., 2002). As a result of this discrimination training, CSX will inhibit the CR that would otherwise be elicited by CSA. Inhibitory associative learning has been a central component in the most influential theories of associative learning (Rescorla and Wagner, 1972; Wagner and Rescorla, 1972; Pearce and Hall, 1980) and has been accounted for by a recent neurocomputational model of associative learning (Mauk and Donegan, 1997). However, very little is known about the neural basis of inhibitory learning.

Eyeblink conditioning has been used as a model system for examining the neural mechanisms of standard (excitatory) clas-

\footnotetext{
Received June 11, 2004; revised Nov. 4, 2004; accepted Dec. 7, 2004. This work was funded by National Institute of Mental Health Grant MH065483.

Correspondence should be addressed to John H. Freeman, Department of Psychology, University of lowa, Seashore Hall, lowa City, IA 52242. E-mail: john-freeman@uiowa.edu. DOI:10.1523/JNEUROSCI.4534-04.2005

Copyright $\odot 2005$ Society for Neuroscience $\quad$ 0270-6474/05/250889-07\$15.00/0
}

sical conditioning and, to a lesser extent, inhibitory conditioning (Mis, 1977; Berthier and Moore, 1980; Moore et al., 1980; Yeo et al.,1983; Blazis and Moore, 1991; Freeman and Nicholson, 1999; Nicholson and Freeman, 2002; Nolan et al., 2002; Christian and Thompson, 2003). Excitatory conditioning requires the intermediate cerebellum including the interpositus nucleus, anterior lobe, hemispheric lobule VI, and perhaps other cortical regions (Christian and Thompson, 2003). Overwhelming evidence from studies using lesions, unit recording, electrical stimulation, and reversible inactivation indicates that the memory that is necessary for excitatory eyeblink conditioning is stored in the cerebellum (Christian and Thompson, 2003). The most convincing evidence supporting the idea of memory storage in the cerebellum comes from studies that used reversible inactivation of the cerebellum and efferent premotor nuclei. Inactivation of the cerebellar nuclei that are ipsilateral to the trained eye with muscimol blocks acquisition of eyeblink conditioning in rabbits (Krupa et al., 1993; Hardiman et al., 1996; Krupa and Thompson, 1997). Muscimol inactivation of the cerebellum also prevents savings, indicating that no learning occurred during inactivation. Inactivation of efferent nuclei or the superior cerebellar peduncle prevents the expression of CRs during training but has no effect on CR production after the inactivation sessions (Krupa et al., 1993; Krupa and Thompson, 1995). The muscimol inactivation studies indicate that the neural plasticity underlying excitatory conditioning is established within the cerebellum and the efferent systems are necessary for motor performance.

The present study used muscimol inactivation of the cerebellum to compare the neural mechanisms underlying excitatory and inhibitory eyeblink conditioning in rats. The first experiment examined the effects of muscimol infusions into the ipsilateral 
anterior interpositus nucleus on acquisition of excitatory conditioning. The second experiment examined the effects of muscimol infusions into the anterior interpositus nucleus on acquisition of inhibitory conditioning. The third experiment examined the extent of muscimol inactivation in the cerebellum and brainstem using 2-deoxy-D-glucose (2-DG) autoradiography.

\section{Materials and Methods}

\section{Experiment 1: effects of muscimol inactivation of the ipsilateral} cerebellum on excitatory eyeblink conditioning

Excitatory eyeblink conditioning in rodents is thought to depend on the cerebellum (Skelton, 1988; Freeman and Nicholson, 1999, 2000; Nicholson and Freeman, 2002, 2003a,b, 2004). However, there are no published reports on the effects of reversible inactivation of the cerebellum on acquisition of eyeblink conditioning in rodents. In addition, many investigators believe that rodents require both cerebellar hemispheres for conditioning (Skelton, 1988; Chen et al., 1996, 1999; Lee and Kim, 2004), but that belief has never been examined systematically. The only evidence against bilateral control of eyeblink conditioning in rodents is that early aspiration lesions of the cerebellar deep nuclei in infant rats abolish conditioning when the rats are more mature (Freeman et al., 1995). The goal of experiment 1 was to determine whether inactivation of the cerebellar hemisphere that is ipsilateral to the trained eye with muscimol blocks acquisition of excitatory conditioning in rats. In phase 1 , the rats were given muscimol (MUS) or saline (SAL) infusions before each of four training sessions with standard excitatory conditioning procedures (see below, Conditioning procedure). A second control group was given saline infusions and exposure to the training apparatus, but no stimuli were presented. All three groups were then given $4 \mathrm{~d}$ of excitatory conditioning with no infusions (phase 2). In phase 3 , all three groups were given muscimol infusions followed by a single session of excitatory conditioning. The context exposure (CE) group was necessary to control for the effects of exposure to the apparatus in the muscimol group, which could influence performance during phase 2 and affect the ability to detect savings or the absence of savings. Context exposure during muscimol inactivation could facilitate conditioning in phase 2, producing what appears to be savings (Hardiman et al., 1996; Krupa and Thompson, 1997). Although unlikely, context exposure could also impair conditioning in phase 2.

Subjects. The subjects were 19 male Long-Evans rats (250-400 g). The rats were housed in the animal colony in Spence Laboratories at the University of Iowa (Iowa City, IA). All rats were maintained on a $12 \mathrm{~h}$ light/dark cycle with light onset at 7:00 A.M. and given ad libitum access to food and water.

Surgery. One week before training, rats were removed from their home cages and anesthetized with an intraperitoneal injection of sodium pentobarbital $(60 \mathrm{mg} / \mathrm{kg})$. To reduce respiratory tract secretions and excess salivation during anesthesia, rats were given injections of atropine sulfate $(0.45 \mathrm{mg} / \mathrm{kg})$. At the onset of anesthesia, the rats were fitted with differential electromyograph (EMG) electrodes that were implanted in the left orbicularis oculi muscle in the upper eyelid. The reference electrode was attached to a stainless steel skull screw. The EMG electrode leads terminated in gold pins in a plastic connector. A bipolar stimulating electrode (Plastics One, Roanoke, VA) for delivering the shock US was implanted subdermally, caudal to the left eye. A 23 gauge guide cannula was implanted $0.5 \mathrm{~mm}$ dorsal to the left anterior interpositus nucleus. A 30 gauge stylet was inserted in the guide cannula. The stereotaxic coordinates taken from bregma were $11.5 \mathrm{~mm}$ posterior, $2.3 \mathrm{~mm}$ lateral, and 5.2 $\mathrm{mm}$ ventral to the skull surface. The plastic connector housing the EMG electrode leads, the bipolar stimulating electrode, the guide cannula, and two skull screws were secured to the skull with dental acrylic (Dentsply International, York, PA). Animals were maintained on $0.006 \%$ Sulfatrim (Alpharma, Baltimore, MD) in water for $4 \mathrm{~d}$ after surgery.

Muscimol infusion procedure. Before the muscimol infusions, a stylet was removed from the guide cannula and replaced with a 30 gauge infusion cannula. The infusion cannula was connected to polyethylene tubing ( $\mathrm{PE} 10 ; 110-120 \mathrm{~cm}$ ), which was connected to a $10 \mu$ l gas tight syringe (Hamilton, Reno, NV). The syringe was placed in an infusion pump
(Harvard Apparatus, Holliston, MA), and $1.0 \mu \mathrm{l}$ of muscimol (10 nmol) was infused at a rate of $30 \mu \mathrm{l} / \mathrm{h}$. The tubing connected to the infusion cannula was cut and sealed with candle wax. The infusion cannula remained in place for the duration of the experimental session.

Apparatus. The conditioning apparatus consisted of four small-animal sound-attenuating chambers (BRS/LVE, Laurel, MD). Within each sound-attenuating chamber was a small-animal operant chamber (BRS/ $\mathrm{LVE}$ ), in which the rats were kept during conditioning. One wall of the operant chamber was fitted with two speakers that independently produce tones of up to $120 \mathrm{~dB}$ (sound pressure level) with a frequency range of $\sim 1000-9000 \mathrm{~Hz}$. The back wall of the sound-attenuating chamber was equipped with a small light. An exhaust fan on one of the walls provided a $65 \mathrm{~dB}$ masking noise. The $\mathrm{CS}$ used in training was a $2000 \mathrm{~Hz}$ pure tone ( $85 \mathrm{~dB}$; range in conditioning chamber, $83-87 \mathrm{~dB}$ ). The electrode leads from the rat's headstage were connected to peripheral equipment by lightweight cables that allowed the rat to move freely during conditioning. A desktop computer was connected to the peripheral equipment. Computer software controlled the delivery of stimuli and the recording of eyelid EMG activity (JSA Designs, Raleigh, NC). One circuit permitted the delivery of a shock stimulus through a stimulus isolator (model 365A; World Precision Instruments, Sarasota, FL). Another circuit amplified differentially (gain, 2000; sampling rate, $250 \mathrm{~Hz}$ ), filtered (500-5000 $\mathrm{Hz}$ ), and integrated (time constant, $20 \mathrm{~ms}$ ) EMG activity. The intensity of the shock US was set at two times threshold for eliciting a discrete eyeblink (range of final current intensity, 2-3 mA; $60 \mathrm{~Hz}$; constant current) (Skelton, 1988).

Conditioning procedure. The rats were allowed to adapt to the training environment for $10 \mathrm{~min}$ before each training and testing session. The SAL $(n=5)$ and MUS $(n=8)$ groups were given saline or muscimol infusions into the anterior interpositus nucleus $20 \mathrm{~min}$ before each of four sessions of classical delay eyeblink conditioning procedures (100 trials per day over $4 \mathrm{~d}$; pseudorandom distribution of intertrial intervals between 18 and $42 \mathrm{~s}$ that averaged $30 \mathrm{~s}$ ), in which a $300 \mathrm{~ms}$ tone CS coterminated with a $25 \mathrm{~ms}$ shock US, yielding an interstimulus interval of $275 \mathrm{~ms}$. The $\mathrm{CE}(n=6)$ group was given saline infusions and exposure to the training apparatus, but no stimuli were presented. The values relayed to the computer software from the EMG integrator are units representing voltage values of integrated EMG activity sampled every $2.5 \mathrm{~ms}$ with a time constant of $20 \mathrm{~ms}$. The CR threshold was set at $0.4 \mathrm{~V}$ above the amplified and integrated EMG activity at baseline [the DC offset $+(0.4$ $\mathrm{V} / 2000 / 0.0025 \mathrm{~s})=$ DC offset $+0.08 \mathrm{~V}$ ]. The EMG baseline was usually zero (except for the DC offset), because the orbicularis oculi muscle does not exhibit spontaneous or tonic activity (Hesslow, 1994; Pellegrini and Evinger, 1997). Integrated EMG responses exceeding the threshold value during the first $80 \mathrm{~ms}$ of the CS period were considered startle responses to the tone CS; responses that exceeded the threshold value during the last $220 \mathrm{~ms}$ of the CS were considered CRs; responses that crossed the threshold after US onset were defined as URs.

Histology. After training, the rats were killed with a lethal injection of sodium pentobarbital $(150 \mathrm{mg} / \mathrm{kg})$ and transcardially perfused with 100 $\mathrm{ml}$ of physiological saline followed by $300 \mathrm{ml}$ of $3 \%$ formalin. After perfusion, the brains were postfixed in the same fixative for a minimum of $24 \mathrm{~h}$ and subsequently sectioned at $50 \mu \mathrm{m}$ with a sliding microtome. Sections were then stained with cresyl violet. The locations of the tips of the infusion cannula were confirmed by examining serial sections.

\section{Experiment 2: effects of muscimol inactivation of the ipsilateral} cerebellum on inhibitory eyeblink conditioning

Neurons in the anterior interpositus nucleus of the rat exhibit activity profiles that correlate with the acquisition and expression of conditioned inhibition (Freeman and Nicholson, 1999; Nicholson and Freeman, 2002). During acquisition, the activity of anterior interpositus neurons develops a neuronal discrimination in which activity during the excitatory stimulus (CSA) becomes greater than the activity during the compound stimulus (CSA and CSX), as the rat learns to respond to CSA and suppress responding to the CSA/CSX compound. After robust discrimination has been established, interpositus neurons show elevated activity during the CSA but suppressed activity during the CSX in a summation test (Nicholson and Freeman, 2002). The cerebellar neuronal correlates 
of conditioned inhibition suggest that it might be acquired by the cerebellum. The goal of experiment 2 was to determine whether inactivation of the cerebellar hemisphere that is ipsilateral to the conditioned eye would block acquisition of conditioned inhibition. In phase 1 , the rats were trained to a criterion of $80 \%$ CRs on 2 consecutive days. In phase 2 , the rats were given either saline or muscimol infusions in the anterior interpositus nucleus before each of five conditioned inhibition training sessions (see below, Conditioning procedure). Phase 3 involved five additional conditioned inhibition training sessions. Phase 4 was a retardation test in which the CSX was trained as an excitatory stimulus. In the retardation test, acquisition of CRs to CSX is impaired relative to control conditions if CSX was established as an inhibitory stimulus during training (phases 2 and 3).

Subjects. The subjects were 13 male Long-Evans rats (250-400 g). All other information regarding the subjects was the same as in experiment 1.

Surgery, muscimol infusion procedure, apparatus, and histology. The methods regarding surgery, muscimol infusions, the apparatus, and histology were the same as in experiment 1.

Conditioning procedure. As in experiment 1, the rats were adapted to the training environment for $10 \mathrm{~min}$ before each training and testing session. The first phase of training in experiment 2 consisted of excitatory conditioning using the same procedures as in experiment 1 , except that the tone was $500 \mathrm{~ms}$ in duration, and conditioning continued until each rat reached a performance criterion of at least $80 \%$ CRs during two consecutive training sessions. During phase 2, rats were given either saline $(n=6)$ or muscimol $(n=7) 30 \mathrm{~min}$ before each of five daily conditioned inhibition training sessions. The conditioned inhibition procedure consisted of 50 paired trials with the tone CS used in phase 1 followed by the US and 50 trials with the tone CS and a light CS presented simultaneously without the US. The conditioned inhibition procedure was designed to establish the light CS as an inhibitory stimulus (Freeman and Nicholson, 1999; Nicholson and Freeman, 2002; Nolan et al., 2002). During phase 3 , conditioned inhibition training continued in the absence of infusions. In previous studies, a different stimulus was used as the reinforced CS in phases 1 and 2 to allow for a summation test after discrimination training (Rescorla, 1969). The same tone was used in phases 1-3 of experiment 2 to establish a baseline of CRs in phase 1 that would be maintained in phase 3 after muscimol inactivation in phase 2 . The baseline of CRs after muscimol inactivation was necessary for detecting discrimination learning in phase 3 that might be established during muscimol inactivation in phase 2 . Phase 4 was the retardation test, consisting of two 100-trial sessions in which the light CS was paired with the US. If the light CS has been established as a conditioned inhibitor, the rats should exhibit fewer CRs during training in phase 4 than control rats given excitatory training with one CS and then transferred to excitatory conditioning using a new CS (Nicholson and Freeman, 2002). In experiment 2, the critical issue for the retardation test is whether the groups given muscimol or saline during training show equivalent responding.

\section{Experiment 3: extent of muscimol inactivation within the} cerebellum and brainstem assessed with 2-DG autoradiography The extent of muscimol induced inactivation within the cerebellum can be assessed indirectly by examining the spread of labeled muscimol. However, the critical issue is the extent of direct and indirect cellular inactivation produced by muscimol, which can be assessed by unit recording or imaging labeled glucose utilization (Martin and Ghez, 1999). Experiment 3 was designed to determine the extent of muscimol inactivation within the cerebellum and brainstem by examining 2-DG uptake after muscimol or saline infusions into the anterior interpositus nucleus with the same infusion procedure and dosage as used in experiments 1 and 2.

Subjects. The subjects were five male Long-Evans rats (250-400 g). All other information regarding the subjects was the same as in experiment 1 .

Surgery and muscimol infusion procedure. The methods regarding surgery and muscimol infusions were the same as in experiments 1 and 2.

2-DG procedure and analysis. As in experiments 1 and 2, rats received a muscimol $(n=3)$ or saline $(n=2)$ infusion into the anterior interpositus nucleus. Twenty minutes after the infusion, each rat received an intraperitoneal injection of a glucose analog, 2-DG radiolabeled with ${ }^{14} \mathrm{C}(20$ $\mu \mathrm{Ci} / 100 \mathrm{~g}$ of body weight; American Radiolabeled Chemicals, St. Louis,

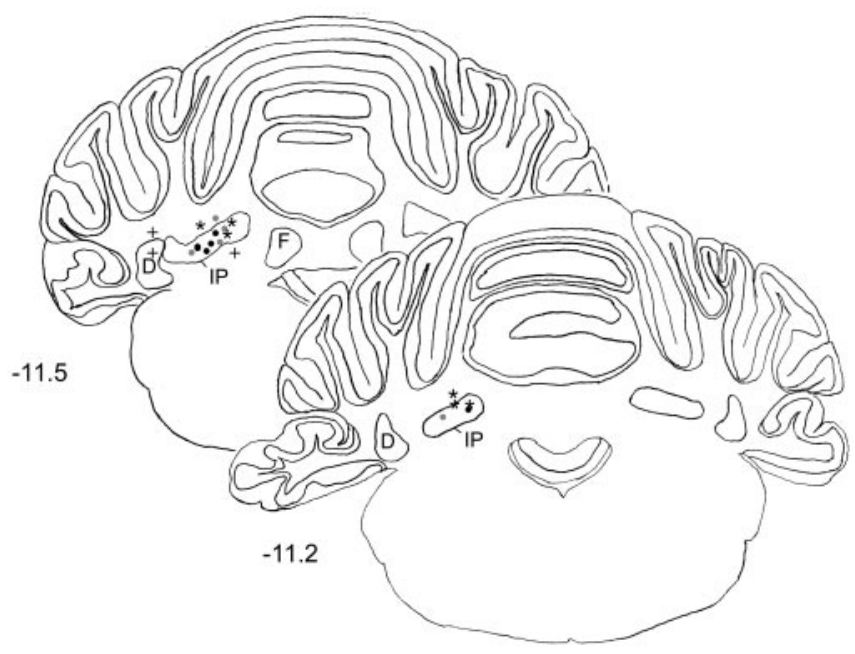

Figure 1. Coronal sections of the rat cerebellum depicting the cannula placements in experiment 1. The cannula placements for the groups given muscimol (black dots) or saline (gray dots) during training were in or just dorsal to the anterior interpositus nucleus (IP). The cannula placements for the group given context exposure (asterisks) were also in or just above the anterior interpositus nucleus. The + symbols indicate the cannula placements that were classified as misses. D, Dentate nucleus; F, fastigial nucleus.

MO) in $0.3 \mathrm{ml}$ of sterile saline. After the 2-DG injection, the rats were allowed to rest in their home cage during the 45 min of 2-DG uptake. Rats were then decapitated, and brains were quickly removed, frozen at $-37^{\circ} \mathrm{C}$, and stored at $-80^{\circ} \mathrm{C}$. The brains were sectioned on a cryostat at $40 \mu \mathrm{m}$, and every section was kept. Sections were quick dried on glass coverslips, glued on poster board, and exposed to ${ }^{14} \mathrm{C}$ phosphor imaging screens for $36 \mathrm{~h}$ along with ${ }^{14} \mathrm{C}$ standards (Amersham Biosciences, Arlington Heights, IL). The screens were read and digitized by a phosphor imaging system (Cyclone Storage Phosphor system; PerkinElmer Life Sciences, Emeryville, CA). Individual calibration curves were calculated based on the absolute gray levels of the ${ }^{14} \mathrm{C}$ standards on each film. Subsequent densitometric measures were then automatically converted to units of nanoCuries per gram.

\section{Results}

\section{Experiment 1}

Figure 1 displays the cannula placements in coronal sections of the cerebellum. The placements of the control groups and the muscimol group were in or just dorsal to the anterior interpositus nucleus, except for three placements. One of the missed placements was ventral to the anterior interpositus nucleus, and the other two were placed in and dorsal to the dentate nucleus.

Muscimol infusion into the anterior interpositus nucleus prevented acquisition of CRs (Fig. 2, sessions 1-4). Acquisition after the infusion sessions was nearly identical in the MUS group during training and the CE control group (sessions 5-8). Moreover, the rates of acquisition for the MUS, CE, and SAL groups did not differ. The absence of a difference between postdrug sessions in the MUS group relative to acquisition in the two control groups indicates that the MUS group did not acquire associative conditioning during initial training (sessions 1-4). All three groups showed nearly complete suppression of CRs during the final muscimol test (session 9), indicating that the cannula placements in all three groups were equivalent.

Repeated-measures ANOVA on the CR percentage data during initial acquisition for the SAL and MUS groups (sessions 1-4) revealed an interaction of the group and session factors $\left(F_{(3,24)}=\right.$ 28.741; $p<0.0001)$. Post hoc tests indicated that the SAL group produced more CRs than the MUS group during all four sessions 


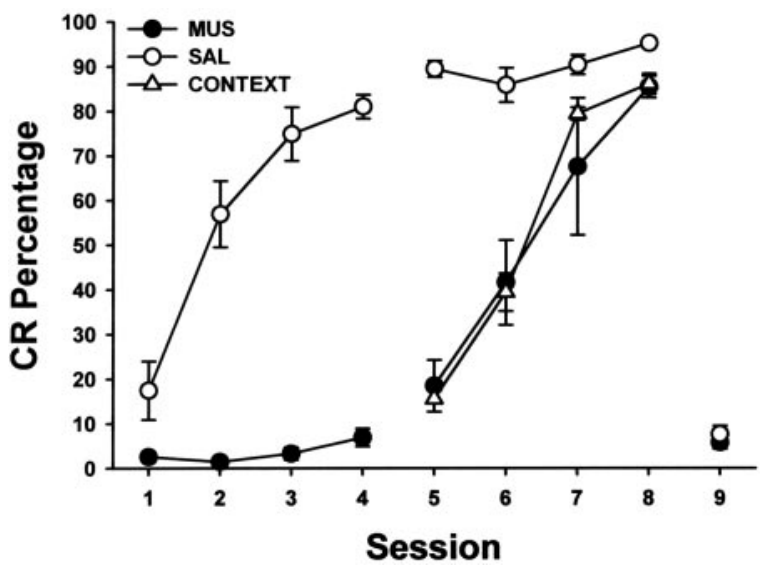

Figure 2. Mean \pm SE CR percentage for rats given MUS or SAL infusions into the anterior interpositus nucleus before sessions $1-4$. A second control group was given saline infusions into the anterior interpositus nucleus and exposed to the training context during sessions $1-4$. All three groups were given additional training during sessions 5-8 with no infusions. Muscimol was infused into the anterior interpositus nucleus in all three groups before training session 9 .

(all comparisons, $p<0.05$ ). It is also evident that magnitude of the difference increased across sessions. Analysis of CR percentage during the second phase of training for all three groups (sessions 5-8) revealed a significant interaction of the group and session factors $\left(F_{(6,39)}=14.75 ; p<0.0001\right)$. Post hoc test indicated that the percentage of CRs in the SAL group was higher than in the MUS and CE groups during sessions 5 and 6 (all comparisons, $p<0.05$ ). The SAL group also exhibited a higher percentage of CRs than the MUS group during session 7 ( $p<0.05)$. The $\mathrm{CE}$ and MUS groups did not differ during sessions $5-8$.

Savings was assessed statistically by comparing the percentage, amplitude, and peak latencies of CRs for the first four acquisition sessions in the SAL (sessions 1-4) and CE (sessions 5-8) groups with the four postdrug sessions in the MUS group (sessions 5-8) with repeated-measures ANOVA. There were no significant group effects or interactions for analyses of CR percentage, amplitude, or peak latency. All three analyses revealed significant effects of the sessions factor (CR percentage, $F_{(3,39)}=106.54, p<$ 0.0001; CR amplitude, $F_{(3,39)}=11.54, p<0.0001$; CR peak latency, $\left.F_{(3,39)}=7.38, p<0.0001\right)$, which reflected the acquisition of CRs across sessions in all three groups.

Three of the rats in the MUS group had cannula placements that were classified as misses based on histological analysis. The group with missed cannula placements (Fig. 3) exhibited complete suppression of CRs during muscimol inactivation (sessions $1-4)$ but then showed substantial savings during the postdrug acquisition sessions (sessions 5-8). Savings was indicated by a higher percentage of CRs in the missed cannula group during sessions 5 and 6 relative to the CE group (Fig. 3). The performance of the group with missed cannula placements indicates that the highest concentration of muscimol must be infused into the anterior interpositus nucleus to completely block conditioning. Infusions of muscimol into the dentate and perhaps the posterior interpositus nucleus block CR performance but do not block learning.

\section{Experiment 2}

The SAL and MUS groups acquired initial excitatory conditioning at the same rate and reached the same level of responding by the end of phase 1 training (Fig. 4, sessions 1-5). During phase 2, the SAL group initially responded at high levels to the reinforced

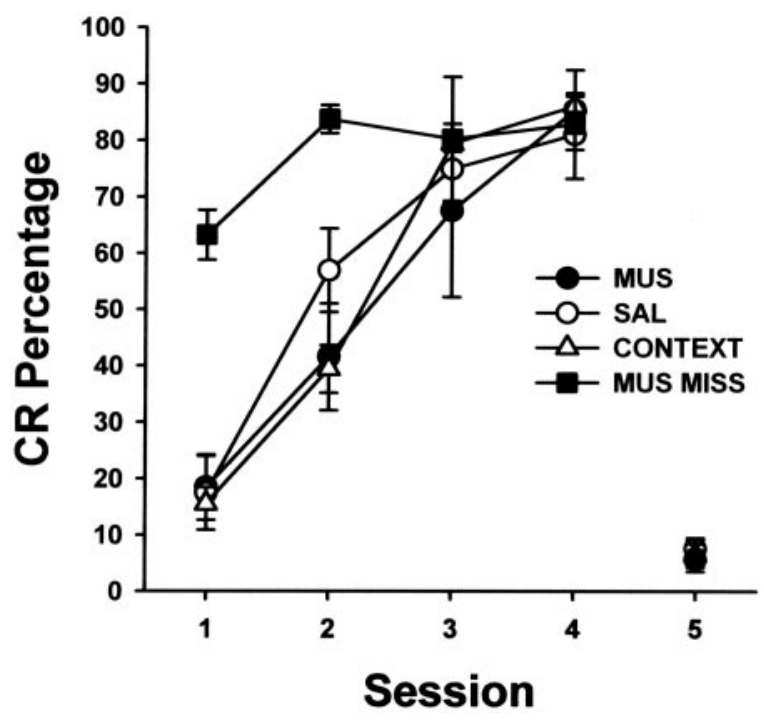

Figure 3. Mean $\pm S E C R$ percentage during the postinfusion sessions for rats given context exposure, muscimol with accurate or missed (MUS MISS) cannula placements, and during the initial training sessions for the group given saline. The higher percentage of CRs in the MUS MISS group on session 1 relative to the CONTEXT and SAL groups indicates savings after initial training with muscimol inactivation. The CR percentage for all of the groups during the posttraining muscimol test is displayed on session 5 .

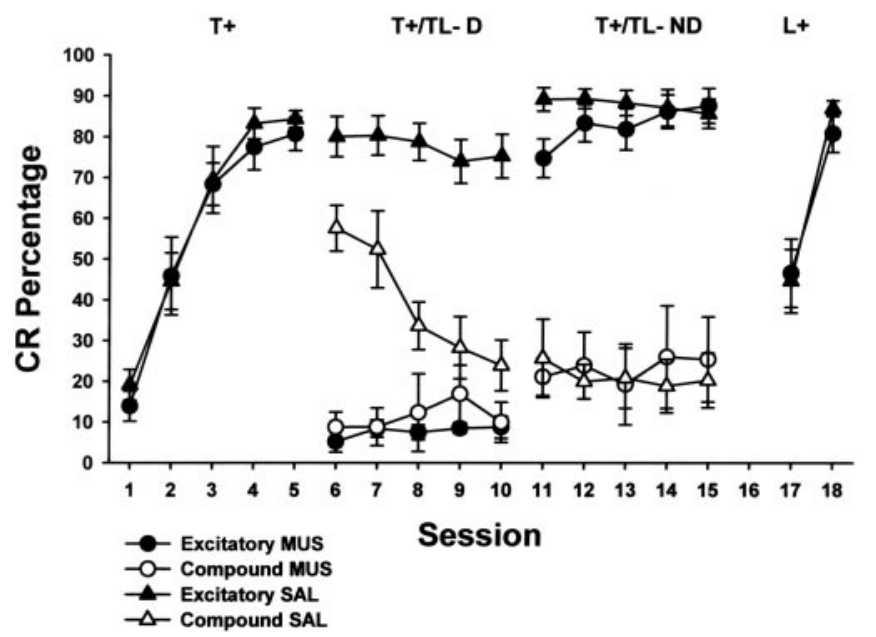

Figure 4. Mean $\pm S E C R$ percentage for rats given saline (triangles) or muscimol (circles) during conditioned inhibition training. Phase 1 of training involved excitatory conditioning with a tone conditioned stimulus. Phases 2 and 3 consisted of 100 -trial sessions $(6-15)$ of conditioned inhibition training, which involved excitatory conditioning trials as in phase 1 and trials with a simultaneous stimulus compound of the tone and a light stimulus, which was not paired with the unconditioned stimulus ( $\mathrm{T}+/ \mathrm{TL}-$ ). The percentage of (Rs occurring during excitatory trials and compound stimulus trials is depicted by black and white symbols, respectively. The final training session was a retardation test in which the light was paired with the unconditioned stimulus ( $L+)$. D, Drug; ND, no drug.

tone CS $(\mathrm{T}+)$ and the unreinforced tone/light compound stimulus $(\mathrm{TL}-)$ but exhibited progressively stronger suppression of responding to $\mathrm{TL}-$ (Fig. 4, sessions 6-10). In contrast, CR performance in the MUS group was suppressed during both $\mathrm{T}+$ and $\mathrm{TL}-$ trials throughout phase 2 training (Fig. 4, sessions 6-10). However, when $\mathrm{T}+$ and $\mathrm{TL}-$ discrimination training continued in phase 3 , both groups exhibited substantial discrimination. The magnitude of discrimination was very similar in the SAL and MUS groups, indicating complete retention of both the excitatory conditioning and discrimination despite the suppression of 


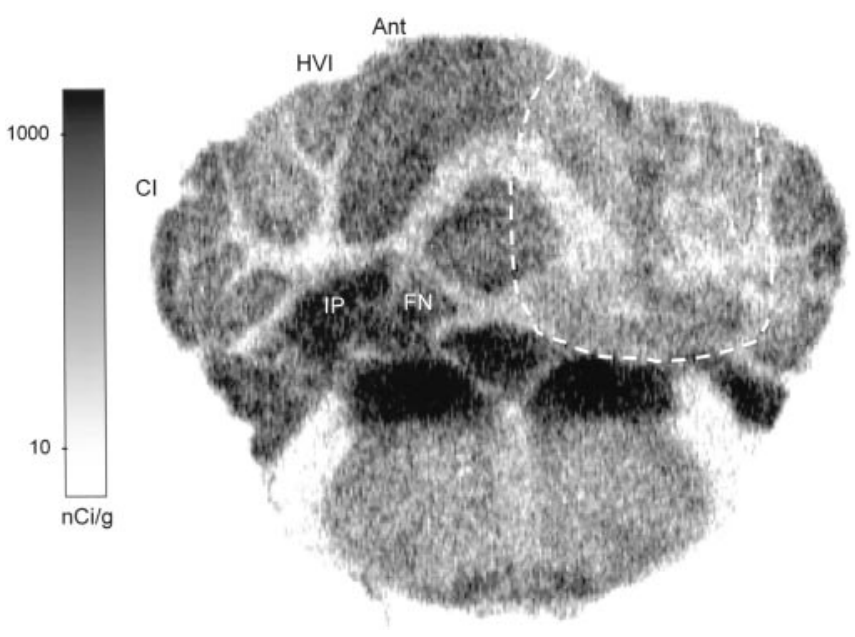

Figure 5. An autoradiograph showing uptake of 2-DG radiolabeled with ${ }^{14} \mathrm{C}$ (black indicating strongest 2-DG uptake). This example demonstrates the area of inactivation after injection of muscimol ( $10 \mathrm{nmol})$ through a cannula centered in the dorsal anterior interpositus nucleus. The white dotted line indicates the boundary of change in 2-DG uptake on the side injected with muscimol. Areas of inactivation include the fastigial nucleus (FN), interposed nuclei (IP), lateral anterior lobe (Ant), crus I (Cl), rostral crus II, and lobule HVI.

CRs during phase 2. Both groups also showed equivalent performance during the retardation test.

The number of sessions to attain the acquisition criterion during phase 1 was compared with a $t$ test, which revealed no group difference (mean sessions to criterion, $\mathrm{SAL}=5.6, \mathrm{MUS}=5.8$ ). Repeated-measures ANOVA compared the percentage of CRs during the two trial types ( $\mathrm{T}+\mathrm{vs} \mathrm{TL}-$ ) in the SAL and MUS groups during phases 2 and 3 across training sessions. A significant interaction was obtained for the group, trial type, phase, and sessions factors $\left(F_{(4,44)}=3.101 ; p<0.03\right)$. Post hoc tests indicated that the SAL group exhibited a higher percentage of CRs during $\mathrm{T}+$ trials relative to $\mathrm{TL}-$ trials during all of the training sessions in phases 2 and 3 (sessions $6-15$; all comparisons, $p<0.05$ ). In contrast, the MUS group exhibited a higher percentage of CRs on $\mathrm{T}+$ trials relative to $\mathrm{TL}-$ trials during phase 3 (sessions 11-15; all comparisons, $p<0.05$ ) but not during phase 2 (sessions 6-10).

The SAL group also exhibited a significantly higher percentage of CRs during $\mathrm{T}+$ trials relative the MUS groups during the first postdrug training session (session $11 ; p<0.05$ ). The group difference in CR percentage during session 11 raised the possibility that the magnitude of discrimination was lower in the MUS group, which would suggest that MUS treatment during phase 2 caused a slight impairment in inhibitory conditioning. An additional repeated-measures ANOVA examined the magnitude of discrimination (CRs during $\mathrm{T}+$ trial minus CRs during $\mathrm{TL}-$ trials) in the SAL and MUS groups across sessions during phase 3. No group effects were detected in the analysis of discrimination magnitude suggesting that inhibitory conditioning was completely unaffected by MUS during phase 2 training.

No group differences were detected by ANOVA for the retardation test (phase 4, sessions 17 and 18). Both groups showed slowed acquisition relative to appropriate control groups, which produce $\sim 70 \%$ CRs on the first session (Nicholson and Freeman, 2002).

\section{Experiment 3}

Analysis of 2-DG autoradiographs from rats given saline or muscimol indicated that uptake of 2-DG was reduced substantially in the cerebellar hemisphere infused with muscimol (Fig. 5). The area of inactivation included the ipsilateral fastigial nucleus, anterior interpositus nucleus, posterior interpositus nucleus, dentate nucleus, lateral anterior lobe, crus I, rostral crus II, and lobule HVI. The cerebellar nuclei and neighboring cortex in the contralateral hemisphere were unaffected by muscimol (Fig. 5). Rats given saline infusions showed no reduction in 2-DG uptake, with strong bilateral 2-DG uptake in all the deep nuclei, vestibular nuclei, and throughout the gray matter of the cortex. The strongest 2-DG uptake was observed bilaterally in the vestibular, interposed, and cochlear nuclei. No increase in 2-DG uptake was observed in the inferior olive contralateral to the muscimol infusion (Fig. 5). However, muscimol induced increases in inferior olive activity might be observed with higher-resolution metabolic imaging techniques.

\section{Discussion}

Acquisition of excitatory eyeblink conditioning in rats was completely blocked by muscimol inactivation of the cerebellar hemisphere that is ipsilateral to the trained eye (experiment 1 ). The rats given muscimol during $4 \mathrm{~d}$ of training showed no evidence of savings in postdrug training sessions relative to control groups that received equivalent training or context exposure for $4 \mathrm{~d}$ before training (Fig. 2). In contrast, rats given muscimol inactivation of the ipsilateral cerebellar hemisphere acquired conditioned inhibition (experiment 2) (Fig. 4). The eyeblink CRs of rats were suppressed by muscimol inactivation during conditioned inhibition training, but the rats given muscimol exhibited robust discrimination during postdrug training sessions. The magnitude of discrimination during postdrug training in the group given muscimol was as robust as the magnitude of discrimination in the control group, indicating that acquisition of conditioned inhibition was completely unaffected by muscimol inactivation of the ipsilateral cerebellar hemisphere.

The findings of the current study indicate that the cerebellumbased models of conditioned inhibition must be modified. Inactivation of the cerebellar hemisphere that is ipsilateral to the trained eye completely prevented excitatory conditioning but had no effect on conditioned inhibition. The neural mechanisms of conditioned inhibition are, therefore, not occurring in the same tissue as the mechanisms underlying excitatory conditioning. This conclusion is seemingly inconsistent with previous studies that found strong correlations between single unit activity in the ipsilateral anterior interpositus nucleus and the amplitude/ time course of eyelid CRs during conditioned inhibition training and testing (Freeman and Nicholson, 1999; Nicholson and Freeman, 2002). Neurons in the anterior interpositus nucleus show increased activity when CRs are produced and suppressed activity when CRs are inhibited. However, the striking neuronal correlates of conditioned inhibition in the anterior interpositus nucleus (Freeman and Nicholson, 1999; Nicholson and Freeman, 2002) are probably best thought of as correlates of learningspecific plasticity occurring in another neural system. The neuronal inhibition observed in the ipsilateral anterior interpositus nucleus during presentations of inhibitory CSs may be driven by extracerebellar structures that modify cerebellar or pontine activity. For instance, neuronal activity in the red nucleus exhibits a stronger correlation with CR suppression during conditioned inhibition training than neuronal activity in the interpositus nucleus in rabbits (Britton et al., 2000; Brown et al., 2000). Inhibitory learning in the red nucleus could affect eyeblink CRs by directly modifying excitatory input to the facial nucleus or through feedback to the pons. Some of the other potential candidates for precerebellar mediation of conditioned inhibition have 
been eliminated. For instance, lesions of the hippocampus (Solomon, 1977) or neodecortication (Moore et al., 1980; Yeo et al., 1983) do not abolish acquisition or retention of conditioned inhibition. However, stimulation and lesion studies indicate that the nucleus of Darkschewitsch, the interstitial nucleus of Cajal, and the anterior red nucleus play roles in conditioned inhibition (Mis, 1977; Berthier and Moore, 1980; Blazis and Moore, 1991). Current studies are examining precerebellar mechanisms of conditioned inhibition.

The cerebellum-based models of conditioned inhibition would still be partially viable if the cerebellar areas not affected by muscimol in the current study are capable of acquiring inhibitory conditioning independently of the cerebellar areas that are necessary for acquisition of excitatory conditioning. Experiment 3 revealed that most of the vermis and contralateral hemisphere were not affected by the muscimol infusion. The unaffected cerebellar areas may be sufficient for acquisition of conditioned inhibition. Evidence for distributed cerebellar cortical circuitry in eyeblink conditioning can be found in a study that used electrical stimulation of the cerebellar cortical surface to identify eyeblink microzones in cats (Hesslow, 1994). Blink microzones were identified based on the presence of short-latency climbing fiber responses to peripheral stimulation and delayed eyelid EMG activity after cortical stimulation. Multiple eyeblink microzones were found in the $\mathrm{C} 1$ and $\mathrm{C} 3$ zones of the cortex in lobules V, VI, VII, and the rostral paramedian lobule. One implication of these findings is that eyeblink microzones not affected by muscimol infusions in the current study might be capable of acquiring conditioned inhibition. However, a recent study that depleted Purkinje cells throughout the cerebellar cortex using the immunotoxin OX7-saporin found impaired acquisition of conditioned inhibition, but all rats with Purkinje cell depletion acquired robust CR inhibition (Nolan and Freeman, 2005). The findings of Nolan and Freeman (2005) suggest that cerebellar cortical areas spared in the current study might play a role in conditioned inhibition but extracerebellar structures must also be necessary.

The findings of experiment 1 indicate that eyeblink conditioning in rats depends on the cerebellar hemisphere that is ipsilateral to the conditioned eye. This finding is consistent with a previous study that found that early lesions of the rat interpositus nucleus and overlying cortex blocked acquisition of eyeblink conditioning in juvenile rats (Freeman et al., 1995). However, Skelton (1988) noted that a preliminary unpublished study found that rats with unilateral lesions of the cerebellar nuclei were not as impaired on eyeblink conditioning as rats given bilateral lesions of the nuclei. Subsequent studies found that bilateral lesions of the interpositus nucleus block conditioning in mice and rats (Chen et al., 1996, 1999; Lee and Kim, 2004). In contrast to all of the other published reports, a recent study using mice indicated that bilateral lesions of the interpositus nucleus did not abolish CRs (Koekkoek et al., 2003). The lesions in this study were accurately placed in the interpositus nucleus but were partial and may have missed the essential part of the nucleus. The current finding of complete loss of excitatory conditioning with unilateral muscimol inactivation in rats indicates that the deficits in eyeblink conditioning produced by bilateral lesions in previous rodent studies are probably primarily caused by damage in the ipsilateral cerebellum.

The current study was designed to inactivate the cerebellar hemisphere that is ipsilateral to the trained eye. It was not designed to examine the effects of selective inactivation of the anterior interpositus nucleus. Indeed, the muscimol infusion used in the current experiments affected all the ipsilateral cerebellar nu- clei and the neighboring cortical areas (Fig. 5). Moreover, the findings of experiment 1 do not prove that the memory for eyeblink conditioning is stored within the cerebellum in rats, as demonstrated in rabbits (Christian and Thompson, 2003). Additional studies are required to examine the roles of specific cerebellar nuclei and structures that are efferent to the cerebellum in rodent eyeblink conditioning.

In conclusion, the neural mechanisms of eyeblink conditioned excitation and inhibition differ substantially. Excitatory eyeblink conditioning is blocked by muscimol inactivation of the cerebellar hemisphere that is ipsilateral to the conditioned eye. In contrast, conditioned inhibition is completely unaffected by inactivation of the ipsilateral cerebellar hemisphere. Conditioned inhibition probably depends on cerebellar areas that were not inactivated in experiment 2 and precerebellar systems. The goal of current research is to identify the precerebellar and cerebellar cortical systems that are necessary for conditioned inhibition.

\section{References}

Berthier NE, Moore JW (1980) Disrupted conditioned inhibition of the rabbit nictitating membrane response following mesencephalic lesions. Physiol Behav 25:667-673.

Blazis DEJ, Moore JW (1991) Conditioned inhibition of the nictitating membrane response in rabbits following hypothalamic and mesencephalic lesions. Behav Brain Res 46:71-81.

Britton G, Brown TC, Steinmetz JE (2000) Single-unit activity from interpositus nucleus during conditioned inhibition of the eyeblink response. Soc Neurosci Abstr 26:268.7.

Brown TC, Britton G, Steinmetz JE (2000) The role of the red nucleus in conditioned inhibition of the rabbit eyeblink response. Soc Neurosci Abstr 26:267.8.

Chen L, Bao S, Lockard JM, Kim JJ, Thompson RF (1996) Impaired classical eyeblink conditioning in cerebellar lesioned and Purkinje cell degeneration (pcd) mutant mice. J Neurosci 16:2829-2838.

Chen L, Bao S, Thompson RF (1999) Bilateral lesions of the interpositus nucleus completely prevent eyeblink conditioning in Purkinje celldegeneration mutant mice. Behav Neurosci 113:204-210.

Christian KM, Thompson RF (2003) Neural substrates of eyeblink conditioning: acquisition and retention. Learn Mem 10:427-455.

Freeman Jr JH, Nicholson DA (1999) Neuronal activity in the cerebellar interpositus and lateral pontine nuclei during inhibitory classical conditioning of the eyeblink response. Brain Res 833:225-233.

Freeman Jr JH, Nicholson DA (2000) Developmental changes in eye-blink conditioning and neuronal activity in the cerebellar interpositus nucleus. J Neurosci 20:813-819.

Freeman Jr JH, Carter CS, Stanton ME (1995) Early cerebellar lesions impair eyeblink conditioning in developing rats: differential effects of unilateral lesions on postnatal day 10 or 20. Behav Neurosci 109:893-902.

Hardiman MJ, Ramnani N, Yeo CH (1996) Reversible inactivations of the cerebellum with muscimol prevent the acquisition and extinction of conditioned nictitating membrane responses in the rabbit. Exp Brain Res 110:235-247.

Hesslow G (1994) Correspondence between climbing fibre input and motor output in eyeblink-related areas in cat cerebellar cortex. J Physiol (Lond) 476:229-244.

Koekkoek SKE, Hulscher HC, Dortland BR, Hensbroek RA, Elgersma Y, Ruigrok TJH, De Zeeuw CI (2003) Cerebellar LTD and learning-dependent timing of conditioned eyelid responses. Science 301:1736-1739.

Krupa DJ, Thompson RF (1995) Inactivation of the superior cerebellar peduncle blocks expression but not acquisition of the rabbit's classically conditioned eye-blink response. Proc Natl Acad Sci USA 92:5097-5101.

Krupa DJ, Thompson RF (1997) Reversible inactivation of the cerebellar interpositus nucleus completely prevents acquisition of the classically conditioned eye-blink response. Learn Mem 3:545-556.

Krupa DJ, Thompson JK, Thompson RF (1993) Localization of a memory trace in the mammalian brain. Science 260:989-991.

Lee T, Kim JJ (2004) Differential effects of cerebellar, amygdalar, and hippocampal lesions on classical eyeblink conditioning in rats. J Neurosci 24:3242-3250. 
Martin JH, Ghez C (1999) Pharmacological inactivation in the analysis of the central control of movement. J Neurosci Methods 86:145-159.

Mauk MD, Donegan NH (1997) A model of Pavlovian eyelid conditioning based on the synaptic organization of the cerebellum. Learn Mem 3:130-158.

Mis FW (1977) A midbrain-brainstem circuit for conditioned inhibition of the nictitating membrane response in the rabbit (Oryctolagus cuniculus). J Comp Physiol Psychol 91:975-988.

Moore JW, Yeo CH, Oakley DA, Russell IS (1980) Conditioned inhibition of the nictitating membrane response in decorticate rabbits. Behav Brain Res 1:397-409.

Nicholson DA, Freeman Jr JH (2002) Neuronal correlates of conditioned inhibition of the eyeblink response in the anterior interpositus nucleus. Behav Neurosci 116:22-36.

Nicholson DA, Freeman Jr JH (2003a) Addition of inhibition in the olivocerebellar system and the ontogeny of a motor memory. Nat Neurosci 6:532-537.

Nicholson DA, Freeman Jr JH (2003b) Developmental changes in evoked Purkinje cell complex spike responses. J Neurophysiol 90:2349-2357.

Nicholson DA, Freeman Jr JH (2004) Developmental changes in eyeblink conditioning and simple spike activity in the cerebellar cortex. Dev Psychobiol 44:45-57.

Nolan BC, Freeman Jr JH (2005) Purkinje cell loss by OX7-saporin impairs excitatory and inhibitory eyeblink conditioning. Behav Neurosci, in press.

Nolan BC, Nicholson DA, Freeman Jr JH (2002) Blockade of GABA receptors in the interpositus nucleus modulates expression of conditioned ex- citation but not conditioned inhibition of the eyeblink response. Integr Physiol Behav Sci 37:293-310.

Pavlov IP (1927) Conditioned inhibition. In: Conditioned reflexes, Chap 5, pp 68-87. London: Oxford UP.

Pearce JM, Hall G (1980) A model for Pavlovian learning: variations in the effectiveness of conditioned but not of unconditioned stimuli. Psychol Rev 87:532-552.

Pellegrini JJ, Evinger C (1997) Role of cerebellum in adaptive modification of reflex blinks. Learn Mem 4:77-87.

Rescorla RA (1969) Pavlovian conditioned inhibition. Psychol Bull 72:77-94.

Rescorla RA, Wagner AR (1972) A theory of Pavlovian conditioning: variations in the effectiveness of reinforcement and nonreinforcement. In Classical conditioning II: current research and theory (Black AH, Prokasy WF, eds), pp 64-99. New York: Appleton-Century-Crofts.

Skelton RW (1988) Bilateral cerebellar lesions disrupt conditioned eyelid responses in unrestrained rats. Behav Neurosci 102:586-590.

Solomon PR (1977) Role of the hippocampus in blocking and conditioned inhibition of the rabbit's nictitating membrane response. J Comp Physiol Psychol 91:407-417.

Wagner AR, Rescorla RA (1972) Inhibition of Pavlovian conditioning: application of a theory. In: Inhibition and learning (Boakes RA, Halliday MS, eds), pp 301-336. London: Academic.

Yeo CH, Hardiman MJ, Moore JW, Russell IS (1983) Retention of conditioned inhibition of the nictitating membrane response in decorticate rabbits. Behav Brain Res 10:383-392. 